\title{
COMPUTATION OF SURFACE WAVE DISPERSION FOR MULTILAYERED ANISOTROPIC MEDIA
}

\author{
By David G. Harkrider and Don L. Anderson
}

\begin{abstract}
With the program described in this paper it is now possible to compute surface wave dispersion in a solid heterogeneous halfspace containing up to 200 anisotropic layers.

Certain discrepancies in surface wave observations, such as disagreement between Love and Rayleigh wave data and other independent evidence, suggest that anisotropy may be important in some seismological problems. In order to study the effect of anisotropy on surface wave dispersion a program was written for an IBM 7090 computer which will compute dispersion curves and displacements for Rayleigh waves in a layered halfspace in which each layer is transversely isotropic. A simple redefinition of parameters makes it possible to use existing programs to compute Love wave dispersion.
\end{abstract}

\section{INTRODUCTION}

The techniques described in previous papers (Dorman et al., 1960; Press et al., 1961) for computing dispersion of surface waves in multilayered isotropic media have been extended so that each layer may be transversely isotropic with an axis of symmetry perpendicular to the layering. The motivation for this extension is the suggestion (Anderson, 1961) that anisotropy may be responsible for certain discrepancies in surface wave data. These discrepancies include disagreement between Love wave and Rayleigh wave studies of both velocity and particle motion. The basic theory is developed in such a way that direct comparison with Haskell's (1953) original work on isotropic layers is possible at each stage. Final mathematical results are in the form of matrix elements which replace those of Haskell and are arranged in a manner convenient for computation.

\section{RAYLeigh WAVES}

As in the isotropic case (Haskell, 1953; Dorman et al., 1960) the theoretical basis for the computation of Rayleigh type surface wave dispersion in a layered aniso tropic halfspace is the vector equation relating the motion-stress vectors at the top and the bottom of each layer

$$
\mathbf{U}_{m}=D_{m} E^{-1} \mathbf{U}_{m-1}^{*}
$$

where $\mathbf{U}_{m}=\left(\dot{u}_{m} / c, \dot{w}_{m} / c, \sigma_{m}, \tau_{m}\right)$. The quantities $\dot{u}_{m}$ and $\dot{w}_{m}$ are the horizontal and vertical particle velocities associated with Rayleigh type motion. $\sigma_{m}$ and $\tau_{m}$ are the normal and tangential stresses. The asterisk on the $\mathbf{U}_{m-1}^{*}$ vector indicates the side of the $m^{\text {th }}$ layer next to the $(m-1)^{\text {th }}$ layer. $D_{m}$ and $E_{m}$ are given in Anderson (1961).

Since the boundary conditions and the form of the vector equation (1) are the same as for Haskell's (1953) isotropic layer formulation, we can make use of the matrix algebra performed by Haskell in obtaining the period equation. Thus all that needs to be done is to evaluate $E_{m}^{-1}$, which is the inverse of $E_{m}$, and the matrix product $D_{m} E_{m}^{-1}$, and then to give the period equation in terms of the matrix elements which are to replace Haskell's. 
Taking the matrix relation (44) from Anderson (1961) and arranging columns as in Haskell (1953) we have

$$
E_{m}=\left[\begin{array}{cccc}
1 & 0 & 1 & 0 \\
0 & i \gamma_{\mathbf{1}_{m}} & 0 & i \gamma_{2_{m}} \\
i X_{\alpha_{m}} & 0 & i X_{\beta_{m}} & 0 \\
0 & Y_{\alpha_{m}} & 0 & Y_{\beta_{m}}
\end{array}\right]
$$

where

$$
\begin{aligned}
& X_{\alpha_{m}}=C_{m} \gamma_{1_{m}} r_{\alpha_{m}}+i F_{m} \\
& X_{\beta_{m}}=C_{m} \gamma_{2_{m}} r_{\beta_{m}}+i F_{m} \\
& Y_{\alpha_{m}}=L_{m}\left(r_{\alpha_{m}}-i \gamma_{1_{m}}\right) \\
& Y_{\beta_{m}}=L_{m}\left(r_{\beta_{m}}-i \gamma_{2_{m}}\right)
\end{aligned}
$$

The above $E_{m}$ differs from Anderson's (1961) by the common factor $i k$ which has been factored out and by two new quantities $r_{\alpha_{m}}$ and $r_{\beta_{m}}$ defined by the relations

$$
r_{\alpha_{m}}=\frac{\nu_{1_{m}}}{i k} \text { and } \quad r_{\beta_{m}}=\frac{\nu_{2_{m}}}{i k}
$$

which have the same sign criteria as Haskell's (1953) $r_{\alpha_{m}}$ and $r_{\beta_{m}}$ or

$$
\begin{array}{ll}
r_{\alpha_{m}, \beta_{m}}=\left|r_{\alpha_{m}, \beta_{m}}\right| & \text { for values of } c \text { such that } r_{\alpha_{m}, \beta_{m}}^{2}>0 \\
r_{\alpha_{m}, \beta_{m}}=-i\left|r_{\alpha_{m}, \beta_{m}}\right| & \text { for values of } c \text { such that } r_{\alpha_{m}, \beta_{m}}^{2}<0
\end{array}
$$

For the isotropic case $r_{\alpha_{m}, \beta_{m}}$ reduce to Haskell's.

Rearranging Anderson's (1961) equation (46) to be consistent with $E_{m}$ we have

$$
D_{m}=\left[\begin{array}{cccc}
\cos P_{m} & i \sin P_{m} & \cos Q_{m} & i \sin Q_{m} \\
-\gamma_{1_{m}} \sin P_{m} & i \gamma_{1_{m}} \cos P_{m} & -\gamma_{2_{m}} \sin Q_{m} & i \gamma_{2_{m}} \cos Q_{m} \\
i X_{\alpha_{m}} \cos P_{m} & -X_{\alpha_{m}} \sin P_{m} & i X_{\beta_{m}} \cos Q_{m} & -X_{\beta_{m}} \sin Q_{m} \\
i Y_{\alpha_{m}} \sin P_{m} & Y_{\alpha_{m}} \cos P_{m} & i Y_{\beta_{m}} \sin Q_{m} & Y_{\beta_{m}} \cos Q_{m}
\end{array}\right]
$$

where

$$
P_{m}=k r_{\alpha_{m}} d_{m} \text { and } Q_{m}=k r_{\beta_{m}} d_{m}
$$

The inverse of $E_{m}$ is then given by 


$$
E_{m}^{-1}=\left[\begin{array}{cccc}
-a_{m} X_{\beta_{m}} & 0 & -i a_{m} & 0 \\
0 & -i b_{m} Y_{\beta_{m}} & 0 & -b_{m} \gamma_{2_{m}} \\
a_{m} X_{\alpha_{m}} & 0 & i a_{m} & 0 \\
0 & i b_{m} Y_{\alpha_{m}} & 0 & b_{m} \gamma_{1_{m}}
\end{array}\right]
$$

where

$$
a_{m}=\frac{1}{X_{\alpha_{m}}-X_{\beta_{m}}} \quad \text { and } \quad b_{m}=\frac{1}{\gamma_{1_{m}} \bar{Y}_{\beta_{m}}-\gamma_{2_{m}} \overline{Y_{\alpha_{m}}}}
$$

and therefore by (2)

$$
a_{m}=\frac{1}{C_{m}\left(\gamma_{1_{m}} r_{\alpha_{m}}-\gamma_{2_{m}} r_{\beta_{m}}\right)}
$$

and

$$
b_{m}=\frac{1}{L_{m}\left(\gamma_{1_{m}} r_{\beta_{m}}-\gamma_{2_{m}} r_{\alpha_{m}}\right)}
$$

and by Anderson's (1961) equation (13) we have

$$
\begin{aligned}
& \gamma_{1_{m}}=i \frac{r_{\alpha_{m}} G_{m}}{\left(\rho_{m} c^{2}-L_{m}-C_{m} r_{\alpha_{m}}^{2}\right)}=i g_{1 m} r_{\alpha m} \\
& \gamma_{2_{m}}=i \frac{r_{\beta_{m}} G_{m}}{\left(\rho_{m} c^{2}-L_{m}-C_{m} r_{\beta_{m}}^{2}\right)}=i g_{2_{m}} r_{\beta_{m}}
\end{aligned}
$$

which are real or imaginary depending on whether $r_{\alpha_{m}, \beta_{m}}$ are imaginary or real, since $g_{1_{m}, 2_{m}}$ are real for $r_{\alpha_{m}, \beta_{m}}^{2}$ real. From the above we see that $X_{\alpha_{m}}$ and $X_{\beta_{m}}$ are always pure imaginary and therefore $a_{m}$ is also imaginary. Thus we will define the real quantities $X_{\alpha_{m}}^{*}, X_{\beta_{m}}^{*}$ and $a_{m}{ }^{*}$ by

$$
X_{\alpha_{m}}=i X_{\alpha_{m}}^{*}, \quad X_{\beta_{m}}=i X_{\beta_{m}}^{*} \text { and } a_{m}=i a_{m}{ }^{*}
$$

or

$$
a_{m}^{*}=\frac{1}{X_{\theta_{m}}^{*}-X_{\alpha_{m}}^{*}}
$$

Also from the above

$$
\begin{aligned}
& Y_{\alpha_{m}}=\rho_{m} \beta_{m}{ }^{2} r_{\alpha_{m}}^{2}\left(1+g_{1_{m}}\right) \\
& Y_{\beta_{m}}=\rho_{m} \beta_{m}{ }^{2} r_{\beta_{m}}^{2}\left(1+g_{2_{m}}\right)
\end{aligned}
$$


where $\beta_{m}{ }^{2}=L_{m} / \rho_{m} ; \beta_{m}$ being the velocity of horizontally traveling $S V$ motion in the $m^{\text {th }}$ layer.

therefore

$$
b_{m}=-i \frac{1}{\rho_{m} \beta_{m}{ }^{2} r_{\alpha_{m}} r_{\beta_{m}} g_{12_{m}}}
$$

where

$$
g_{12_{m}}=g_{1_{m}}-g_{2_{m}}
$$

Carrying out the matrix multiplication for $\boldsymbol{a}_{m}=D_{m} E_{m}^{-1}$ we have as the elements of this product

$$
\begin{aligned}
& \left(\boldsymbol{a}_{m}\right)_{11}=a_{m}{ }^{*}\left[X_{\boldsymbol{\beta}_{m}}^{*} \cos P_{m}-X_{\alpha_{m}}^{*} \cos Q_{m}\right] \\
& \left(\boldsymbol{a}_{m}\right)_{12}=i\left[-\frac{\left(1+g_{2_{m}}\right)}{g_{12_{m}}}\left(\frac{\sin P_{m}}{r_{\alpha_{m}}}\right)+\frac{\left(1+g_{1_{m}}\right)}{g_{12_{m}}}\left(\frac{\sin Q_{m}}{r_{\beta_{m}}}\right)\right] \\
& \left(\boldsymbol{a}_{m}\right)_{13}=a_{m}{ }^{*}\left[\cos P_{m}-\cos Q_{m}\right] \\
& \left(\boldsymbol{a}_{m}\right)_{14}=i\left[-\frac{g_{2_{m}}}{\rho_{m} \beta_{m}^{2} g_{12_{m}}}\left(\frac{\sin P_{m}}{r_{\alpha_{m}}}\right)+\frac{g_{1_{m}}}{\rho_{m} \beta_{m}^{2} g_{12_{m}}}\left(\frac{\sin Q_{m}}{r_{\beta_{m}}}\right)\right] \\
& \left(\boldsymbol{a}_{m}\right)_{21}=i a_{m}{ }^{*}\left[-X_{\boldsymbol{\beta}_{m}}^{*} g_{1_{m}}\left(r_{\alpha_{m}} \sin P_{m}\right)+X_{\boldsymbol{\alpha}_{m}}^{*} g_{2_{m}}\left(r_{\beta_{m}} \sin Q_{m}\right)\right] \\
& \left(\boldsymbol{a}_{m}\right)_{22}=\frac{g_{1_{m}}\left(1+g_{2_{m}}\right)}{g_{12_{m}}} \cos P_{m}-\frac{g_{2_{m}}\left(1+g_{1_{m}}\right)}{g_{12_{m}}} \cos Q_{m} \\
& \left(\boldsymbol{a}_{m}\right)_{23}=i a_{m}{ }^{*}\left[-g_{1_{m}}\left(r_{\alpha_{m}} \sin P_{m}\right)+g_{2_{m}}\left(r_{\beta_{m}} \sin Q_{m}\right)\right] \\
& \left(\boldsymbol{a}_{m}\right)_{24}=\frac{g_{1_{m}} g_{2_{m}}}{\rho_{m} \beta_{m}^{2} g_{12_{m}}}\left[\cos P_{m}-\cos Q_{m}\right] \\
& \left(\boldsymbol{a}_{m}\right)_{31}=-a_{m}^{*} X_{\alpha_{m}}^{*} X_{\boldsymbol{\beta}_{m}}^{*}\left[\cos P_{m}-\cos Q_{m}\right] \\
& \left(\boldsymbol{a}_{m}\right)_{32}=i\left[X_{\alpha_{m}}^{*} \frac{\left(1+g_{2_{m}}\right)}{g_{12_{m}}}\left(\frac{\sin P_{m}}{r_{\alpha_{m}}}\right)-X_{\beta_{m}}^{*} \frac{\left(1+g_{1_{m}}\right)}{g_{12_{m}}}\left(\frac{\sin Q_{m}}{r_{\beta_{m}}}\right)\right] \\
& \left(a_{m}\right)_{33}=a_{m}^{*}\left[-X_{\alpha_{m}}^{*} \cos P_{m}+X_{\beta_{m}}^{*} \cos Q_{m}\right] \\
& \left(\boldsymbol{a}_{m}\right)_{34}=i\left[X_{\alpha_{m}}^{*} \frac{g_{2_{m}}}{\rho_{m} \beta_{m}{ }^{2} g_{12_{m}}}\left(\frac{\sin P_{m}}{r_{\alpha_{m}}}\right)-X_{\beta_{m}}^{*} \frac{g_{1_{m}}}{\rho_{m} \beta_{m}{ }^{2} g_{12_{m}}}-\left(\frac{\sin Q_{m}}{r_{\beta_{m}}}\right)\right] \\
& \left(\boldsymbol{a}_{m}\right)_{41}=i a_{m}{ }^{*}\left[X_{\beta_{m}}^{*} \rho_{m} \beta_{m}{ }^{2}\left(1+g_{1_{m}}\right)\left(r_{\alpha_{m}} \sin P_{m}\right)-X_{\alpha_{m}}^{*} \rho_{m} \beta_{m}{ }^{2}\left(1+g_{2_{m}}\right)\left(r_{\beta_{m}} \sin Q_{m}\right)\right]
\end{aligned}
$$




$$
\begin{aligned}
& \left(\boldsymbol{a}_{m}\right)_{42}=-\rho_{m}{\beta_{m}}^{2} \frac{\left(1+g_{1_{m}}\right)\left(1+g_{2_{m}}\right)}{g_{12_{m}}}\left[\cos P_{m}-\cos Q_{m}\right] \\
& \left(\boldsymbol{a}_{m}\right)_{43}=i a_{m}{ }^{*} \rho_{m} \beta_{m}{ }^{2}\left[\left(1+g_{1_{m}}\right)\left(r_{\alpha_{m}} \sin P_{m}\right)-\left(1+g_{2_{m}}\right)\left(r_{\beta_{m}} \sin Q_{m}\right)\right] \\
& \left(\boldsymbol{a}_{m}\right)_{44}=-\frac{\left(1+g_{1_{m}}\right)}{g_{12_{m}}} g_{2_{m}} \cos P_{m}+\frac{\left(1+g_{2_{m}}\right)}{g_{12_{m}}} g_{1_{m}} \cos Q_{m}
\end{aligned}
$$

For the isotropic case we have

$$
\begin{aligned}
Y_{\alpha_{m}} & =\rho_{m} c^{2} r_{\alpha_{m}} \gamma_{m} & Y_{\beta_{m}} & =-\frac{\rho_{m}}{r_{\beta_{m}}} c^{2}\left(\gamma_{m}-1\right) \\
X_{\alpha_{m}}^{*} & =-\rho_{m} c^{2}\left(\gamma_{m}-1\right) & X_{\beta_{m}}^{*} & =-\rho_{m} c^{2} \gamma_{m} \\
a_{m}^{*} & =-\frac{1}{\rho_{m} c^{2}} & g_{1_{m}} & =1 \\
g_{2_{m}} & =-\frac{1}{r_{\beta_{m}}^{2}} & g_{12_{m}} & =\frac{c^{2}}{\beta_{m}^{2} r_{\beta_{m}}^{2}}
\end{aligned}
$$

and substituting these values in $\boldsymbol{a}_{m}$ we obtain the $\boldsymbol{a}_{m}$ given by Haskell (1953).

Also the same matrix elements are real or imaginary in this horizontally isotropic case for the $\boldsymbol{a}_{m}$ matrix as in Haskell's pure isotropic case. Now, carrying through the boundary conditions, i.e. continuity of motion-stress vectors across welded solid to solid interfaces, no sources at infinity in the halfspace, and zero normal and tangential stresses at the free surface, we finally obtain as in Haskell

$$
[K N-L M]+[N G-L H][T]=0
$$

where

$$
T=\frac{\sigma_{l}}{\dot{w}_{l} / c}
$$

and $\sigma_{l}$ and $\dot{w}_{l}$ are the normal stress and the vertical displacement respectively at the solid-liquid interface if a surface liquid layer is present. In that case

$$
T=i T^{*}=i \frac{\rho_{l}}{r_{\alpha_{l}}} c^{2} \tan P_{l}
$$

If no surface liquid layer is present then $T=0$ at the free surface and we obtain the same expression as Haskell. In this particular anisotropic case the following quantities are imaginary and are redefined as shown, where $A=\boldsymbol{a}_{n-1} \cdots \boldsymbol{a}_{1}$ 


$$
\begin{aligned}
A_{j k} & =i A_{j k}^{*} \quad \text { where } j+k \text { is an odd integer } \\
L & =i L^{*} \\
M & =i M^{*} \\
G & =i G^{*}
\end{aligned}
$$

and relation (12) for the existence of surface waves becomes

where

$$
F \equiv\left[K N+L^{*} M^{*}\right]-\left[G^{*} N-L^{*} H\right]\left[T^{*}\right]=0
$$

$$
\begin{aligned}
& K=a_{n}{ }^{*} X_{\beta_{n}}^{*}\left|r_{\alpha_{n}}\right| A_{12}^{*}-\frac{\left(1+g_{2_{n}}\right)}{g_{12_{n}}} A_{22}+a_{n}{ }^{*}\left|r_{\alpha_{n}}\right| A_{32}^{*}-\frac{g_{2_{n}}}{\rho_{n} \beta_{n}{ }^{2} g_{12_{n}}} A_{42} \\
& L^{*}=-a_{n}{ }^{*} X_{\beta_{n}}^{*}\left|r_{\alpha_{n}}\right| A_{11}-\frac{\left(1+g_{2_{n}}\right)}{g_{12_{n}}} A_{21}^{*}-a_{n}{ }^{*}\left|r_{\alpha_{n}}\right| A_{z 1}-\frac{g_{2_{n}}}{\rho_{n} \beta_{n}{ }^{2} g_{12_{n}}} A_{41}^{*} \\
& M^{*}=-a_{n}^{*} X_{\alpha_{n}}^{*} A_{12}^{*}-a_{n}^{*} A_{32}^{*}+\frac{\left(1+g_{1_{n}}\right)}{g_{12_{n}\left|r_{\beta_{n}}\right|}} A_{22}+\frac{g_{1_{n}}}{\rho_{n} \beta_{n}^{2} g_{12_{n}}\left|r_{\beta_{n}}\right|} A_{42} \\
& G^{*}=-a_{n}{ }^{*} X_{\beta_{n}}^{*}\left|r_{\alpha_{n}}\right| A_{13}-\frac{\left(1+g_{2_{n}}\right)}{g_{12_{n}}} A_{23}^{*}-a_{n}^{*}\left|r_{\alpha_{n}}\right| A_{33}-\frac{g_{2_{n}}}{\rho_{n} \beta_{n}{ }^{2} g_{12_{n}}} A_{43}^{*} \\
& H=-a_{n}{ }^{*} X_{\alpha_{n}}^{*} A_{13}-a_{n}^{*} A_{33}-\frac{\left(1+g_{1_{n}}\right)}{g_{12_{n}}\left|r_{\beta_{n}}\right|} A_{23}^{*}-\frac{g_{1_{n}}}{\rho_{n} \beta_{n}{ }^{2} g_{12_{n}}\left|r_{\beta_{n}}\right|} A_{43}^{*} \\
& N=-a_{n}^{*} X_{\alpha_{n}}^{*} A_{31}-\frac{\left(1+g_{1_{n}}\right)}{g_{12_{n}}\left|r_{\beta_{n}}\right|} A_{21}^{*}-\frac{g_{1_{n}}}{\rho_{n} \beta_{n}^{2} g_{12_{n}}\left|r_{\beta_{n}}\right|} A_{41}^{*}
\end{aligned}
$$

and where the subscript $n$ represents the halfspace.

Again, for the case of isotropic media, these relations reduce to the ones defined in Haskell (1953).

\section{Love Waves}

It is shown in Anderson (1962) that upon substitution of the following pseudoparameters

$$
d_{m}^{\prime}=\left(\frac{N_{m}}{L_{m}}\right)^{1 / 2} \dot{d}_{m}
$$

and

$$
\mu_{m}{ }^{\prime}=\left(L_{m} \cdot N_{m}\right)^{1 / 2}
$$


into the motion-stress vector equation matrix $\boldsymbol{a}_{m}{ }^{2}$ the matrix is equivalent to the isotropic matrix for Love waves (Haskell, 1953) where $d_{m}$, the $m^{\text {th }}$ layer thickness, is replaced by $d_{m}{ }^{\prime}$ and $\mu_{m_{1}}$ the $m^{\text {th }}$ layer isotropic rigidity, is replaced by $\mu_{m}{ }^{\prime}$. The quantities $N_{m}$ and $L_{m}$ are the $m^{\text {th }}$ layer directional rigidities as defined in Anderson (1961) for horizontally isotropic layers. The isotropic layer is a special case of the above where $N_{m}=L_{m}=\mu_{m}$ and we have $d_{m}{ }^{\prime}=d_{m}$ and $\mu_{m}{ }^{\prime}=\mu_{m}$. Expressions in Haskell's Love formulation containing the isotropic shear velocity are the same in this anisotropic case with shear velocity being replaced by the shear velocity of horizontally traveling $\mathrm{SH}$ waves.

With the substitution of these pseudo-parameters in the halfspace relations, the period equation for Love type surface waves in $(n-1)$ horizontally isotropic layers overlying a horizontally isotropic halfspace is identical with the period equation obtained in Haskell and used as the basis of calculation of Love wave dispersion in the computer program described in Press et al. (1961).

Therefore for the calculation of Love type dispersion in multilayered horizontally isotropic media, one simply substitutes the pseudo-parameters defined above for the individual layer rigidities and thicknesses into a program for calculating isotropic Love wave dispersion based on the Haskell matrix formulation such as the one described in Press et al. (1961).

\section{Method of Computation}

Unfortunately, for the general case of Rayleigh type dispersion, it is not possible to define pseudo-parameters and use a previous program for isotropic layers as in Love dispersion. 'Therefore a new program was written for horizontally isotropic layers. This program is written in FORTRAN for the IBM 7090 and can be used to find the dispersion (phase velocity versus period) and displacement at each interface for Rayleigh type motion in problems involving up to 200 solid horizontally isotropic layers over a solid horizontally isotropic halfspace. In addition, a liquid layer can be inserted at the free surface above this array. Since the case of a three-dimensionally isotropic layer is a special case of the horizontally isotropic layer where the vertical and horizontal elastic constants are equal, it is possible to use this program in problems involving a mixture of isotropic and horizontally isotropic layers.

The general computational procedure is to find the zeroes of the $F$ function (14), which is a function of phase velocity, wave number, and the four elastic constants of the layers. The program flow is similar to the program described in Press et al. (1961), in that the zeroes of the $F$ function are determined by initially specifying the phase velocity and a trial value of the wave number $k$. The elements of the $\boldsymbol{a}_{m}$ matrix are formed for each layer and then multiplied by the matrix for the layer below it, starting with the solid layer nearest the free surface. After the matrix product for all the layers have been calculated, the quantities for the halfspace are calculated and combined with the layer product matrix elements to obtain the quantities defined in (15). If a liquid surface layer is present, $T^{*}$ is calculated next. These numerical values are then combined to obtain a numerical value of the $F$ function (14). The $F$ function is formed so that its value is positive for $k$ 's less than the root in the gravest mode. New trial values of $k$ (of increasing or decreasing size 
depending on the sign of the initial $F$ value) specified by $\Delta k$, which is an input parameter, are used to calculate new values of the $F$ function until a change in sign of $F$ is detected. Linear interpolation and extrapolation are then repeatedly used to find smaller $F$ values until $k$ 's of different $F$ sign are within the precision interval desired. The resulting interpolated value of $k$ is the root to the required precision.

The root $k$ is then used to recalculate the elements used to evaluate $F$. These new elements are combined to form the horizontal to vertical particle velocity amplitude ratio at the free surface or, if a liquid surface layer is present, to form the same ratio in the solid at the liquid solid interface. The group velocity is computed by numerical differentiation of the phase velocity values. This is done by perturbing the phase velocity by a small amount and then finding a new $k$ root to be used in the difference relation. If displacements at depth are desired, the program will now calculate the particle velocity ratios at each interface normalized to the surface vertical velocity by iterative use of (1) using the previously calculated horizontal to vertical surface ratio as the initial value.

The phase velocity is decremented by an input specified amount, and then a new $k$ using the previous root as a trial $k$ and group velocity are calculated by repeating the above procedure. The order of normal mode calculated is controlled by an input which determines the sign of $F$ for $k$ 's less than the root and the initial trial $k$ and $\Delta k$ being in a region such that the roof finding process gives the desired mode root.

\section{Some Programming Problems}

As in Press et al. (1961), numbers of the order of $\exp \left(k \sum_{i=1}^{n-1} d_{i}\right)$ where $d_{i}$ is the $i^{\text {th }}$ layer thickness, are involved in the calculation of $F$. Therefore as the phase velocity decreases and the values of $k$ increase, the larger root values of $k$ will lead to machine overflow, even in floating point arithmetic, if the total number of layers remains constant. Thus when the elements in the $F$ factor become large enough to cause a machine overflow, the program will automatically reduce the number of layers used in the calculation, starting with the halfspace and proceeding toward the free surface. This process will continue to eliminate layers with shear velocity greater than the phase velocity until the elements in the $F$ function no longer cause an overflow. Then before proceeding to calculate the new root, the program will recalculate the root for the preceding phase velocity in order to verify that no loss in precision of $k$ was caused by layer reduction.

For isotropic layers, the quantities $r_{\alpha_{m}}$ and $r_{\beta_{m}}$ are either real or imaginary for all real values of phase velocity. However, as will be discussed in a later paper, for even very slight departures from isotropy, there exist regions of real phase velocity for which $r_{\alpha_{m}, \beta_{m}}$ will be complex for layers in the array. Unfortunately these regions may oceur for phase velocities which would be part of the real dispersion curve if all the layers were completely isotropic. Computing complex dispersion for more than one layer is extremely difficult and therefore was not attempted in this program. Therefore, when a phase velocity is such that $r_{\alpha_{m}, \beta_{m}}$ becomes complex for one of the layers, the machine automatically converts this layer and all other layers in the array for which $r_{\alpha_{m}}, \beta_{m}$ is complex to equivalent isotropic layers. The 
new isotropic layers use the horizontal compressional and the horizontal SV velocities for their compressional and shear velocities respectively. The program then recalculates the root for the preceding phase velocity in order to estimate the degree of error in making the anisotropic layers isotropic. If the error is large the case can be re-run with a greater number of layers approximating a given velocity gradient.

Unlike the program described in Press et al. (1961), there is no double precision

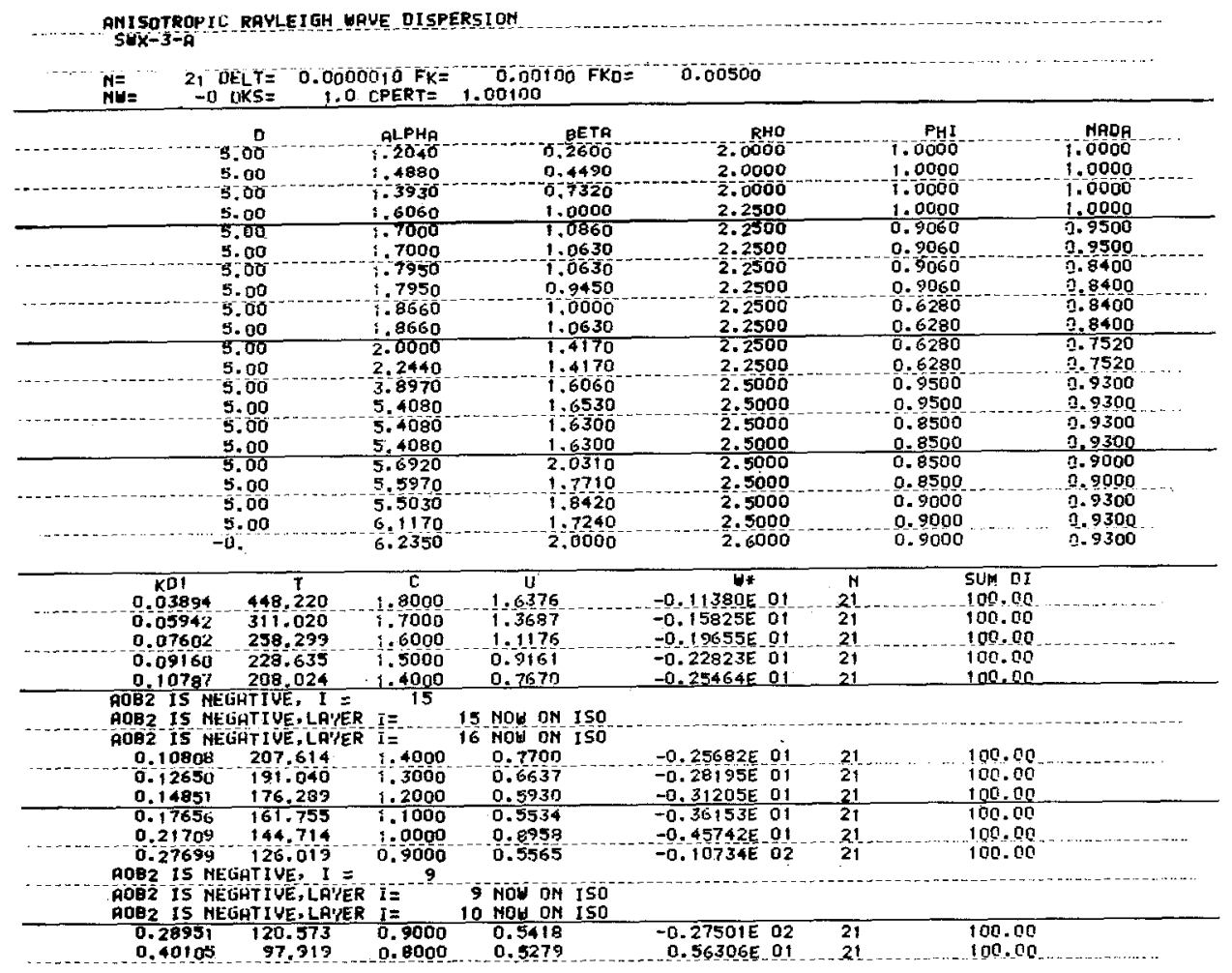

LAST OUTPUT FOR THIS DAIA

FIG. 1. Output format for anisotropic Rayleigh wave dispersion calculation.

feature built into the program to handle a loss of significance in root differences for group velocity calculations. This error may occur in regions where $k$ is a slowly varying function of phase velocity and can be corrected by increasing the phase velocity perturbation factor used in the group velocity calculation. Therefore all group velocities in regions of slowly varying $k$ or period should be graphically verified.

\section{RESULTS}

For 20 horizontally isotropic layers overlying a halfspace, the program will calculate 10 values of phase and group velocity in roughly 2 minutes.

Figure 1 shows a sample calculation for 20 layers. At two phase velocities $r_{\alpha_{m}}, \beta_{m}$ 
became complex and for the rest of the calculations the layers with complex $r_{\alpha_{m}}, \varepsilon_{m}$ were assumed isotropic. These were layers 15 and 16 at a phase velocity of 1.4 , and layers 9 and 10 at a phase velocity of 0.9 . Figure 2 shows the results for a displacement at depth calculation.

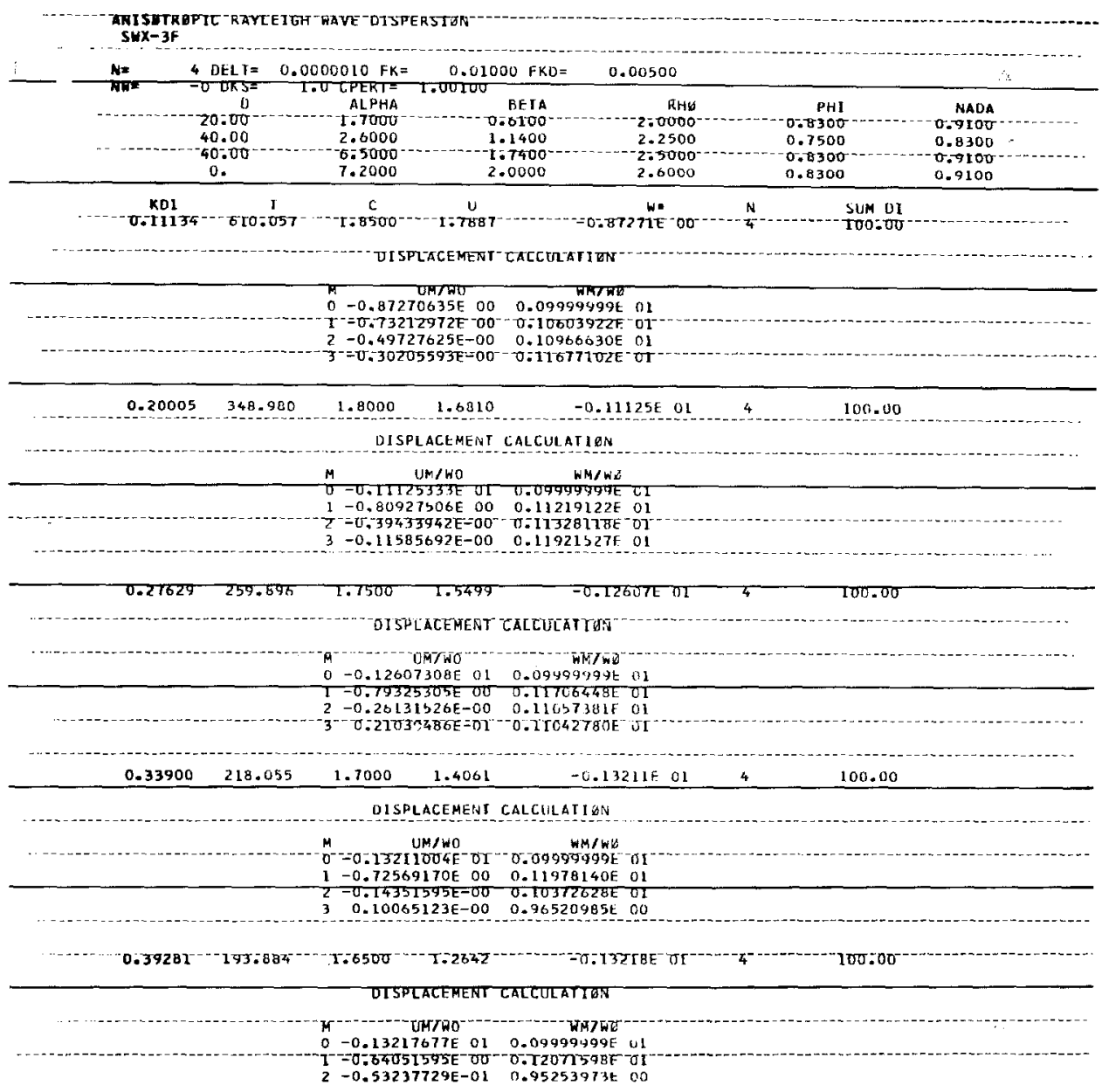

FIG. 2. Output format of displacement at depth calculation.

The notation is as follows:

D Thickness

ALPHA Horizontal traveling compressional wave velocity

BETA Horizontal traveling $S V$ wave velocity

RHO Density

PHI Squared ratio of vertical to horizontal traveling compressional wave velocity

NADA Squared ratio of $45^{\circ}$ to horizontal traveling compressional wave velocity

KD Dimensionless wave number with respect to top layer 
$\mathrm{T} \quad$ Period in same time units as associated with layer velocities

$\mathrm{C}, \mathrm{U} \quad$ Phase and group velocity in same units as layer velocities

$W^{*} \quad$ Ratio of horizontal to vertical displacement at top of solid nearest free surface

$\mathrm{N} \quad$ Number of layers including halfspace used in computation. Change in $\mathrm{N}$ indicates automatic layer reduction is operative.

M Interface at which displacement is calculated starting at free surface. Repeated number indicates liquid-solid interface.

$\mathrm{UM} /$ WO, Horizontal and vertical displacement at $M^{\text {th }}$ interface with respect $\mathrm{WM} / \mathrm{WO}$ to vertical displacement at free surface (FORTRAN floating point notation)

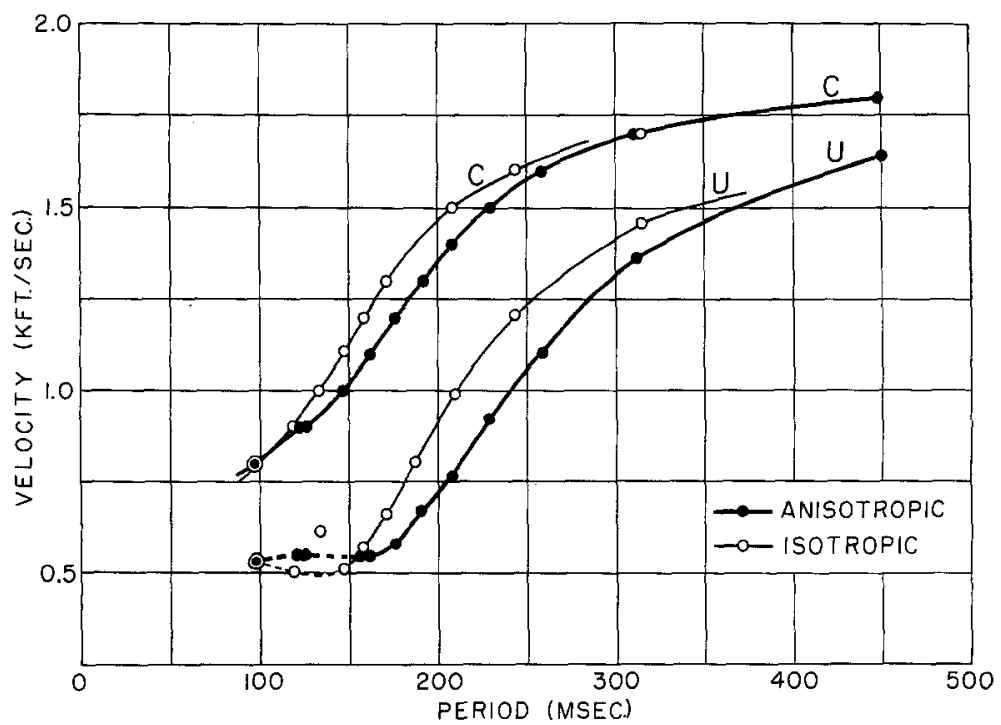

FrG. 3. Sample effect of horizontal isotropy on Rayleigh wave dispersion curves.

The dispersion curves in figure 3 illustrate the effect of horizontal isotropy for the case computed in figure 1. The anisotropic curve is drawn from the values in figure 1, and the isotropic curve is for the same case with the exception that the anisotropy factors PHI and NADA are equal to unity. Although the program assumption of making layers 9 and 10 isotropic appears to give a large error of approximately $6 \mathrm{sec}$ at a phase velocity of 0.9 in figure 1, the actual effect on the phase velocity curve is slight as seen in figure 3. Note the scattering of group velocity points near the group velocity minimum due to a lack of significance in the root $k$ differences used in the group velocity calculation.

\section{ACKNOWLEDGMENTS}

This paper represents research conducted at the Seismological Laboratory of the California Institute of Technology under Contract No. AF-49(638)910 of the Air Force Technical Applications Center as part of the Advanced Research Projects Agency project VELA. 


\section{REFERENCES}

Anderson, Don L.

1961. "Elastic Wave Propagation in Layered Anisotropic Media," Jour. Geophys. Res., 66, 2953-2963.

1962. "Love Wave Propagation in Multilayered Anisotropic Media," Geophysics (in press). Dorman, J., M. Ewing, and J. Oliver

1960. "Study of Shear Velocity Distribution in the Upper Mantle by Mantle Rayleigh Waves," Bull. Seism. Soc. Am., 50, 87-115.

Haskell, N. A.

1953. "Dispersion of Surface Waves on Multilayered Media," Bull. Seism. Soc. Am., 43, 17-34.

Press, Frank, David Harkrider, and C. A. Seafeldt

1961. "A Fast Convenient Program for Computation of Surface Wave Dispersion Curves in Multilayered Media," Bull. Seism. Soc. Am., 51: 495-502.

Seismological Laboratory,

California Institute of Technology,

Pasadena, California

(Division of the Geological Sciences, contribution no. 1057)

Manuscript received October 19, 1961. 